\title{
CONCURRENT DISCRIMINATION LEARNING OF MONKEYS AFTER HIPPOCAMPAL, ENTORHINAL, OR FORNIX LESIONS ${ }^{1}$
}

\author{
MARK MOSS, ${ }^{2}$ HELEN MAHUT, AND STUART ZOLA-MORGAN ${ }^{3}$ \\ Department of Psychology, Northeastern University, Boston, Massachusetts, 02115
}

\begin{abstract}
Ablations of anterior inferotemporal cortex in monkeys are known to impair learning when discriminations between members of several pairs of objects are taught concurrently. This deficit has been attributed to a loss of visual mnemonic functions. But ablations of hippocampus have also been shown to impair retention, and this impairment transcends the visual modality. Therefore, in the first of two experiments, we compared the behavioral effects of inferotemporal cortical lesions with those of either hippocampus, entorhinal area, or fornix, using a visual concurrent discrimination task. Monkeys with either hippocampal or entorhinal ablations were impaired, while those with fornix sections were not. However, ablations of hippocampus included inadvertent damage of the inferotemporal cortex. Therefore, in the second experiment, behavioral effects of inferotemporal lesions were compared with those of hippocampus (without additional inferotemporal damage) on the concurrent task in both visual and tactual modalities. In the visual mode, monkeys with hippocampal removals were as impaired as those with inferotemporal ablations. In the tactual mode, however, hippocampal, but not inferotemporal, ablations were followed by a deficit.

Our results, taken together with other existing evidence, emphasize the role of the hippocampus in mediating associative learning in more than one modality. These results, obtained with non-human primates, are in line with clinical findings.
\end{abstract}

It is firmly established that the anterior part of the inferotemporal neocortex in the monkey is a critical focus for visual discrimination learning (for reviews, see Gross, 1973; Dean, 1976). In particular, removal of this area produces a severe deficit on the concurrent discrimination task which involves the presentation of eight pairs of objects in an intermingled fashion until the animals learn to discriminate every pair. However, operated monkeys are not impaired in learning the discrimination of similar object pairs when these are presented individually. Since, with concurrent presentation, associations between reward and given stimulus objects have to be

\footnotetext{
${ }^{1}$ We wish to thank Dr. M. Mishkin for his help in preparing monkeys with hippocampal resections without inferotemporal damage and Dr. D. Pandya for his help with the surgery and histological examination of entorhinal resections. This work was supported by grants to $H$. M. from the National Science Foundation (BNS 77-173112), the Foundations' Fund for Research in Psychiatry (G 67364), and a grant-in-aid from the Biomedical Research Support, Northeastern University. The preparation of this manuscript was supported by Public Health Service, National Institute of Child Health and Human Development Grant HD 08135-05.

${ }^{2}$ Present address: Harvard Neurological Unit, Beth Israel Hospital, Boston, MA 02215.

${ }^{3}$ Present address: Veterans Administration Hospital, University of California, School of Medicine, La Jolla, CA 92093.
}

acquired in the presence of proactive and retroactive interpair interference, the impairment has been interpreted as a disturbance in the associative mnemonic aspect of discrimination learning (Iwai and Mishkin, 1968; Cowey and Gross, 1970).

Although deficits on a visual task similar to the concurrent discrimination task have been seen in monkeys with combined removals of hippocampus and amygdala (Correll and Scoville, 1965), attempts to find impairments in associative learning following selective removals of the hippocampus have been generally disappointing (Iversen, 1976). Recent findings in our laboratory, however, revealed that the hippocampus may, in fact, be implicated in memory functions of monkeys. With a task in which monkeys had either to learn a reversal of an easy discrimination acquired the day before or to demonstrate retention of a discrimination learned the day before (Task AB), hippocampal resections were followed by a deficit on retention, but not on reversal, davs. This selective impairment was present in both visual and tactual modes. Furthermore, monkeys with resections of the hippocampal formation were impaired on a task which only required retention of object discriminations after either 1-, 24-, or 48-hr intervals (Mahut et al., 1979, 1981).

Hence, behavioral evidence suggests that both the anterior inferotemporal cortex and hippocampus may be implicated in memory and learning. This need not be 
surprising in view of close anatomical connections between the two structures: Rostral portions of the inferotemporal cortex project to the rhinal cortical areas (Whitlock and Nauta, 1956; Jones and Powell, 1970; Moss, 1974; Van Hoesen and Pandya, 1975a) which, in turn, project to the hippocampus (Van Hoesen and Pandya, 1975b).

In light of both behavioral and anatomical evidence, it appeared plausible that the hippocampus might be involved in the mediation of associative learning attributed to the inferotemporal neocortex. Accordingly, in experiment 1 , the performance of monkeys with removals of either hippocampal formation or anterior inferotemporal cortex was compared to that of normal, control monkeys on a visual concurrent discrimination task. In view of the pivotal anatomical role of rhinal cortical areas, we also assessed the effects of entorhinal lesions. A group of monkeys with sections of the fornix was included since such sections disrupt a major afferent and efferent pathway of the hippocampus and, thus, provide an important anatomical and functional control for the effects of entorhinal removals. In a parallel experiment, 1a, one of us (S. Z. -M.) tested five normal, control monkeys and five monkeys with bilateral fornix transections.

\section{EXPERIMENTS 1 AND 1A}

\section{Materials and Methods}

\section{Subjects}

Subjects were 30 Macaca mulatta (13 female, 17 male). Of these, 20 took part in experiment 1 and 10 took part in experiment 1a. In experiment 1 , the intended lesions were: anterior inferotemporal cortex, AIT $(n=5)$; hippocampal formation, HA $(n=3)$; entorhinal cortex, Ent $(n=4)$; and columns of the fornix, Fx $(n=3)$. Five monkeys served as an unoperated, control group, N. In experiment 1a, the intended lesion was a bilateral transection of the columns of the fornix, $\mathrm{Fx}^{\prime}(n=5)$, and five monkeys served as an unoperated control group, $\mathrm{Na}$. All animals were housed individually and were maintained on a diet of Purina Chow, supplemented daily with vitamins and fruit. Feeding took place twice a day, the last approximately $14 \mathrm{hr}$ before testing.

In experiment 1 , all monkeys were experimentally naive when first tested on three visual discrimination tasks (brightness, hue, and pattern). However, before the administration of the concurrent task, they had been trained on a series of spatial and object discrimination reversals as well as on two object discrimination retention tasks (Mahut et al., 1981). In experiment 1a, monkeys had been trained only on spatial and object discrimination reversal tasks.

\section{Surgery}

Detailed descriptions of surgical procedures were given previously (Mahut et al., 1981). Briefly, they were as follows: Anterior inferotemporal lesions were performed by subpial aspiration of the cortex corresponding to the rostral portion of area TE of von Bonin and Bailey (1947). Hippocampal ablations were performed by making an incision through the caudal tip of the rhinal sulcus.
Once visualized, the hippocampus was resected by aspiration. Entorhinal ablations were made by subpial aspiration of the cortex medial to the posterior two-thirds of the rhinal sulcus, corresponding to Brodmann's areas 28a and 28b (cited by Van Hoesen and Pandya, 1975a). Fornix sections were made by making a small incision in the anterior portion of the corpus callosum and by raising and cutting the columns with a small nerve hook at a level just caudal to the septum.

\section{Histological Verification of Lesions}

Detailed histological methods and descriptions of the lesions were given previously (Mahut et al., 1981). They can be summarized as follows: In experiment 1 , all five AIT monkeys sustained complete bilateral ablations limited to the intended area of removal. The three HA monkeys sustained either total or near total bilateral resections of the hippocampal formation (Ammon's horn, dentate gyrus, prosubiculum, and subiculum). Sparing of the anterior third of the hippocampus was found only in one monkey (HA-2). All three animals sustained inadvertent bilateral damage of the anterior inferotemporal neocortex and of areas TF-TH of von Bonin and Bailey (1947). This damage was moderate in monkey HA-1 and extensive in monkeys HA-2 and HA-3. There was no direct involvement of either amygdala or temporal stem in any of the three animals. However, fiber degeneration was observed in the temporal stem of each HA monkey, corresponding to the presence and extent of anterior inferotemporal cortical damage. The three monkeys in the entorhinal group had complete bilateral removals of entorhinal cortex and, in one of these (Ent-3), there was also a small, bilateral lesion of anterior inferotemporal cortex. In the fourth monkey, the classical entorhinal areas were spared. Instead, this monkey (TF-TH) had a bilateral lesion of the cortical areas $\mathrm{TF}$ and $\mathrm{TH}$ and, to a smaller extent, of the ventral aspects of areas OA and $\mathrm{OB}$ of von Bonin and Bailey (1947). In the fornix group, all monkeys had complete transections of both columns of the fornix. In one (Fx-3), the callosal incision was more caudal than in the other two monkeys and, as a result, this animal also sustained a section of the dorsal hippocampal commissure. In experiment $1 \mathrm{a}$, all five $\mathrm{Fx}^{\prime}$ monkeys had sustained complete bilateral transections of the fornix columns.

\section{Apparatus and Procedure}

Testing took place in a modified Wisconsin General Testing Apparatus. The experimenter sat behind a oneway screen facing a tray containing two food wells, 36 $\mathrm{cm}$, center to center. Between trials, the tray was concealed from the animals by an opaque door. The following discrimination tasks were given.

\section{Individual visual discriminations}

The three discrimination tasks were brightness (blackwhite), hue (red-green), and pattern (yellow cross versus outline of yellow square on black background, matched for relative yellow and black areas). Whenever possible, the first stimulus object of each pair was positive (baited) for half the animals in each group and negative (un- 
baited) for the other half. The locations of the two objects over the left and right food wells varied in a predetermined order (Gellerman, 1933) and raisins, or small pieces of apple, were used as bait. A non-correction procedure was followed and 30 trials a day were given, 6 days a week, until a learning criterion of 90 correct responses in 100 consecutive trials was met or for a maximum of 800 trials.

\section{Concurrent discrimination I}

As in the studies of Iwai and Mishkin (1968) and of Cowey and Gross (1970), eight pairs of junk objects were used. The pairs, mounted on 3-inch square gray plaques, were presented in an intermingled fashion during each testing session so that all eight discriminations had to be learned simultaneously. Specifically, on each trial, one pair of objects was presented and, over the course of each testing session of 40 trials, any given pair appeared five times. The left-right position of the positive and negative members of each pair and the order in which the pairs appeared across the 40 trials on a given day were based on a predetermined sequence. Testing was continued until a learning criterion of 39 correct responses in 40 consecutive trials was met in one session or for a maximum of 1500 trials.

\section{Concurrent discrimination II}

Whereas concurrent discrimination I was designed to assess simultaneous learning of eight pairs of objects, this version of the task was used to determine the effect of presenting concurrently pairs that had been previously learned individually. New pairs of objects were used and the task consisted of four stages (a to d), similar to those described by Cowey and Gross (1970).

Stage $a$. Five pairs of objects were used, each presented for 50 trials a day, until a learning criterion of 45 correct responses was reached in one session. A new pair was presented only after learning criterion had been reached with a preceding pair.

Stage $b$. All animals were tested for retention of the five pairs that they had learned in stage a. The pairs were presented individually in the same order as before, each for 50 trials a day, until the original learning criterion was met with each pair.

Stage $c$. The five pairs of objects learned in the two previous stages were now presented concurrently. Within a daily session of 50 trials, therefore, each of the five pairs was presented 10 times in a predetermined order. Training was continued until a learning criterion of 49 correct responses in 50 consecutive trials was reached either within one session or over 2 days.

Stage $d$. Upon completion of stage c, a concurrent discrimination task was presented in which, in addition to the five familiar pairs, there were five new pairs of objects. Thus, in a daily session of 50 trials, each pair appeared five times. The animals were trained to a learning criterion of 49 correct responses in 50 consecutive trials either within one session or over 2 days.

Monkeys in experiment la were trained only on the concurrent discrimination I task.

In experiment 1 , the data obtained by the five groups of monkeys (N, AIT, HA, Ent, Fx) were subjected to a one-way analysis of variance (Kruskal-Wallis), followed by individual comparisons between groups using the Mann-Whitney $U$ test. The learning scores obtained by one monkey with bilateral removals of areas TF-TH are tabulated separately and were not included in the statistical comparisons. In experiment 1a, the data obtained by normal and operated groups were compared by means of the Mann-Whitney $U$ test. Two-tailed levels of significance were used, unless otherwise specified. Inspection of the data showed that differences between groups based on errors were paralleled by those based on trials, therefore, only statistical comparisons based on errors will be reported.

\section{Results}

\section{Experiment 1: Individual discrimination tashs}

The results obtained in the first two visual discrimination tasks are summarized in Table I.

Brightness. Overall comparison among the five groups of monkeys yielded an $H$ value of 10.33 , significant at better than the 0.05 level of confidence. Normal error scores ranged from 4 to 25 . Monkeys with either hippocampal (HA) or entorhinal (Ent) lesions made more errors than did normal monkeys ( $U$ values $=0 ; p$ values $=0.036$ ). Although AIT monkeys were not significantly impaired as a group, three of the five animals obtained higher error scores than any of the normal, control monkeys. Monkey TF-TH made 50 errors, a higher score than that obtained by any of the normal monkeys.

Hue. No significant group difference was found ( $H=$ $5.37 ; p>0.05)$.

Pattern. The results are presented in Table II. Overall group comparison yielded an $H$ value of 13.22 , significant at better than the 0.02 level of confidence. As expected, monkeys in group AIT made significantly more errors than did those in the normal, control group $(U=0 ; p=$ $0.008)$. Also, they performed significantly worse than either HA or Fx groups $(U=0 ; p=0.036$, for both comparisons). Group HA made significantly more errors than the normal group ( $U=0 ; p=0.036)$, but only one monkey with entorhinal lesions (Ent-3) failed to learn the task within the limits of testing ( 800 trials). It should be noted that each monkey in group $\mathrm{HA}$, and monkey Ent-3, had additional damage of the anterior inferotemporal neocortex.

\section{Concurrent discrimination I}

Individual data for all animals are shown in Table II. Overall group comparison yielded an $H$ value of 10.65 , significant at better than the 0.05 level of confidence. Groups HA and Ent obtained significantly higher error scores than did the normal, control group ( $U=0 ; p=$ 0.036 , for both comparisons). Monkey TF-TH made more errors than any of the normal monkeys. Monkeys with AIT damage were not significantly impaired as a group, but three of the five animals obtained elevated learning scores. Possible reasons for the unusually good performance of monkeys AIT- 1 and 2 will be considered later. 
TABLE I

Group mean errors for brightness and hue discrimination tasks

\begin{tabular}{lccc}
\hline \multicolumn{1}{c}{ Group } & $\mathrm{N}$ & Brightness & Hue \\
\hline Normal & \multicolumn{3}{c}{ Experiment I } \\
Anterior inferotemporal & 5 & 12.0 & 4.2 \\
Hippocampal & 5 & 57.4 & 26.0 \\
Entorhinal & 3 & $44.3^{a}$ & 9.0 \\
$\quad$ TF-TH & 3 & $75.3^{a}$ & 32.0 \\
Fornix & 1 & 50 & 12 \\
& 3 & 16.0 & 3.0 \\
Normal & & & \\
Anterior inferotemporal & 4 & Experiment 2 & \\
Hippocampal & 5 & 28.2 & 3.3 \\
& 7 & 35.3 & 13.0 \\
\hline
\end{tabular}

${ }^{a} U=0 ; p=0.036$

In sharp contrast to monkeys in groups $\mathrm{HA}$ and Ent, those with fornix sections were not only unimpaired, but two of the three animals obtained better learning scores than did the most efficient normal, control monkey. Interestingly, the third animal (Fx-3), with an appreciably higher error score, was the one with additional damage of the hippocampal commissure.

\section{Performance patterns}

In the hope of uncovering some underlying coherent pattern of performance which could further distinguish operated from normal monkeys, the data were inspected in the following manner. For each monkey, the number of errors made with each of the eight pairs of objects was tabulated, day by day, throughout all the testing sessions taken by that animal to learn the task. It will be remembered that each of the eight pairs appeared five times during each testing session of 40 trials. We arbitrarily said that a pair had been learned when, on 2 consecutive days, no more than one error was made with that pair. This allowed us to distinguish two main learning patterns: (1) At least six pairs learned within 2 to 3 days (clustered) or (2) learning of the eight pairs occurred throughout the testing sessions (distributed). A mixture of the two patterns was encountered only in three of the 20 monkeys (Ent-3, AIT-2, and Fx-3). The clustering described in pattern 1 was frequently found close to the last testing session, just before the animals met the stringent learning criterion of 39 correct in 40 trials required for the completion of the task.

The performance of normal, control monkeys fell equally into the distributed $(n=2)$ and clustered $(n=3)$ categories. The performance of the two monkeys with fornix sections, without accompanying damage of the hippocampal commissure, was only of the clustered type, as was that of the two unimpaired, AIT-1 and AIT-2, monkeys. All impaired monkeys showed the distributed pattern. One other feature distinguished the performance of normal and operated, but unimpaired monkeys, from that of operated, but impaired, monkeys. Once the former learned a given pair, not more than one error was made with that pair on subsequent days. In contrast, all operated, impaired monkeys, continued to make few, but consistent, errors for many subsequent sessions.
These informal observations suggest that normal monkeys learned several pairs simultaneously and that, once learned, the discriminations were well retained, allowing speedy acquisition of the remaining pairs. Impaired monkeys tended to learn the eight discriminations successively, with long intervening pauses. It became of interest, therefore, to see at what point they began to experience significantly more difficulty than did normal monkeys in learning successive pairs. This was done by noting the number of daily sessions required by monkeys in a given group to learn their first, second, and each of the remaining six pairs. The results of this analysis are illustrated in Figure 1. There were no significant differences among groups in the number of days required to learn the first five pairs. However, monkeys in the HA, Ent, and AIT groups required significantly more sessions to learn the remaining three pairs ( $U$ values $=0 ; p$ values $=0.036$ ) than did those in either the normal control or Fx groups.

TABLE II

Experiment 1: Performance of monkeys with inferotemporal cortical ablations and those with selective lesions within the hippocampal system on visual pattern and concurrent object discrimination tasks

The symbol, + , denotes failure to reach learning criterion within limits of testing. Animals in the hippocampal and entorhinal groups are coded numerically for size of lesion (smallest, 1), with low variability in the size of the lesion in group AIT. Animals within each group are ordered with respect to errors on concurrent task.

\begin{tabular}{cccccc}
\hline & \multicolumn{2}{c}{ Pattern } & & \multicolumn{2}{c}{ Concurrent I } \\
\cline { 2 - 3 } Group & Errors & $\begin{array}{c}\text { \% Correct of } \\
\text { Last } 100 \text { Trials }\end{array}$ & & Trials & Errors \\
\hline Normal & 154 & & & \\
N-1 & 49 & 90 & & 320 & 96 \\
N-2 & 104 & 91 & & 440 & 137 \\
N-3 & 224 & 91 & & 440 & 139 \\
N-4 & 210 & 90 & & 520 & 143 \\
N-5 & & 90 & & 560 & 159
\end{tabular}

Anterior

inferotemporal

AIT-1

AIT-2

$356+$

AIT-3

$360+$

AIT-4

$377+$

AIT-5

403

Hippocampal

HA-1 270+

HA-2

234+

HA-3

$238+$

75

69

$1500+$

$1500+$

Entorhinal

Ent-3 370+

Ent-1

226

Ent-2

117

51

91

91

800

1040

1400

245

TF-TH

100

90

800

215

Fornix

Fx-1

Fx-2

$\Gamma x-3$

92
125
184

91

90

120

37

184

91
281

$389+$

47

48

177

261

316

$485+$ 


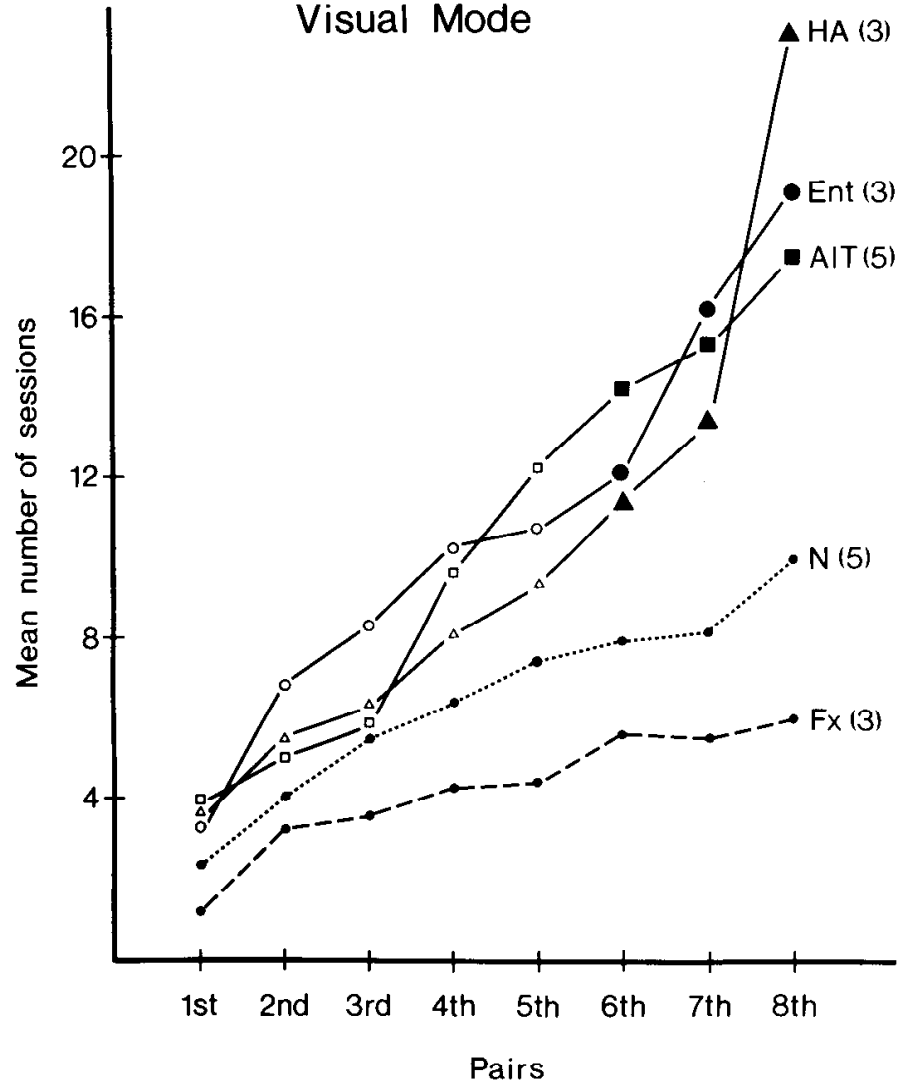

Figure 1. Experiment 1: Rates at which five groups of monkeys learned successively each of the eight discrimination problems presented concurrently. Larger solid symbols indicate significant differences from the normal, control group.

Figure 1 illustrates the performance of all three monkeys in group Fx. However, two monkeys (Fx-1 and Fx2) learned each of the eight pairs significantly faster than did normal monkeys ( $U$ values $=0 ; p$ values $=0.047$, onetailed test). By contrast, monkey $\mathrm{Fx}-3$, with accompanying damage of the hippocampal commissure, and monkey TF-TH needed more sessions than the slowest normal monkey to learn pairs 4 through 8 .

\section{Concurrent discrimination II}

Stage $a$. No significant differences among groups were found in the total number of errors on any of the five discrimination problems. Mean error scores for all five problems ranged between 24.3 and 28.3 for all groups.

Stage $b$. All groups retained the five discriminations learned in stage a with group mean error scores for all five problems ranging from 3.7 to 6.0 .

Stage c. No significant group differences were found in the number of errors to learning criterion when the five object pairs were presented concurrently. Mean error scores ranged from 2.6 to 4.0 , for all groups.

Stage $d$. When five new pairs were presented concurrently with the five original pairs, a significant group difference was found $(H=12.22 ; p<0.02)$. Errors obtained by the normal, control group ranged from 24 to
55, with a mean of 43.8. Groups HA and Ent, with mean errors of 73.0 and 70.3, respectively, made more errors than did normal, control monkeys $(U=0 ; p=0.036$, for both comparisons). Monkey TF-TH obtained an elevated score of 61 errors. Monkeys in group AIT were not significantly impaired as a group, due to normal error scores obtained by monkeys AIT-1 and AIT-2 ( 35 and 43 errors, respectively). However, the errors made by the remaining three animals in the group were elevated and ranged from 63 to 75 . The three monkeys in group $\mathrm{Fx}$, with error scores of 21,24 , and 30 , respectively, performed within normal limits.

There was no significant group difference in the number of errors made with the five old pairs of objects; group mean errors ranged from $6.0(\mathrm{~N})$ to $12.3(\mathrm{HA})$. However, operated monkeys accumulated most of their errors on the five new pairs. Thus, while the mean number of errors with the familiar five pairs ranged between 11.2 (Ent) and 18.6 (AIT) for all groups, those made with five new pairs ranged between 81.4 (AIT) and 88.8 (Ent).

\section{Experiment 1a: Concurrent discrimination I}

No significant group difference was found in the number of errors made on concurrent task I (Table III); monkeys with fornix sections learned the task as readily as did normal, control monkeys. The curve obtained by plotting the mean number of sessions required to learn each of the eight consecutive pairs was almost identical to that obtained by monkeys in group $\mathrm{Fx}$ in experiment 1 (see Fig. 1).

\section{Comments}

At first glance, the results of experiment 1 suggested that impairment on the concurrent discrimination task may result from damage to temporal lobe structures other than the inferotemporal neocortex, and the deficit seen after removals of the entorhinal area strongly supported this notion. Unfortunately, since, in all three HA monkeys, resections of the hippocampus were accompanied by inadvertent bilateral damage of the inferotemporal cortex, a direct assessment of the effects of damage

TABLE III

Experiment Ia: Comparison between the performance of normal monkeys and those with sections of the fornix on visual concurrent discrimination task $I$

\begin{tabular}{ccc}
\hline Group & Trials & Errors \\
\hline Normal & & \\
Na-1 & 320 & 99 \\
$\mathrm{Na}-2$ & 200 & 101 \\
$\mathrm{Na}-3$ & 520 & 129 \\
$\mathrm{Na}-4$ & 440 & 130 \\
$\mathrm{Na}-5$ & 480 & 139 \\
& & \\
Fornix & & \\
Fx'-1 & 480 & 80 \\
$\mathrm{Fx}^{\prime}-2$ & 440 & 97 \\
$\mathrm{Fx}^{\prime}-3$ & 560 & 120 \\
$\mathrm{Fx}^{\prime}-4$ & 400 & 133 \\
$\mathrm{Fx}^{\prime}-5$ & 760 & 159 \\
\hline
\end{tabular}


to the hippocampus alone was not possible. It was clear, however, that, in contrast to monkeys with entorhinal removals, those with transections of the fornix were not impaired.

The finding that not all monkeys with anterior inferotemporal removals were impaired was inconsistent with previous findings (Iwai and Mishkin, 1968; Cowey and Gross, 1970). Yet, the lesions sustained by our AIT animals, both in terms of locus and size, were comparable to those found in the two earlier studies, and they did result in a deficit in visual pattern discrimination. Possibly, extensive intervening practice with a variety of discrimination tasks (see "Subjects") may have been responsible for improved visual function. To test this notion, AIT and normal control groups were retested, after the concurrent task, on the same pattern discrimination task as the one used at the beginning of the sludy (cross versus square). It will be remembered that, initially, all five monkeys in the AIT group had performed at chance level on the last 100 trials of this task (Table II). On retest, however, their impairment was no longer present; all monkeys reached learning criterion within 110 trials and the mean error scores for the normal and AIT groups did not differ significantly from each other (15.6 and 25.8, respectively, for the two groups).

In view of the inconclusive results obtained with monkeys in the hippocampal group and the relative recovery from the effects of surgery of monkeys in the AIT group, new groups of animals were prepared with either anterior inferotemporal or hippocampal removals (experiment 2). This time, to avoid inadvertent damage to the inferotemporal cortex, a different surgical approach to the hippocampus was used and the concurrent discrimination tasks were administered immediately following the three individual visual discriminations (brightness, hue, and pattern).

Since medial temporal lobe damage in patients, when it includes the hippocampus, produces memory deficits not only in visual, but also in auditory and tactual modalities (Milner, 1974), it became of interest to check on possible modality nonspecific effects of hippocampal resections. To this end, after testing in the visual mode, monkeys in the normal, hippocampal, and inferotemporal groups were taught a concurrent discrimination task in the tactual modality.

\section{EXPERIMENT 2}

\section{Materials and Methods}

\section{Subjects and Surgery}

The subjects were 16 experimentally naive Macaca mulatta ( 6 female, 10 male), weighing between 4 and 6 $\mathrm{kg}$ at the time of surgery. They were divided into the following three groups: five monkeys with anterior inferotemporal ablations ( $\left.\mathrm{AIT}^{\prime}\right)$, seven with hippocampal resections (HT), and four serving as an unoperated, control group $\left(\mathrm{N}^{\prime}\right)$.

Anterior inferotemporal lesions were performed using the same procedure as that used in experiment 1 . For hippocampal resections, a large triangular craniotomy was made over the lateral portion of the temporal lobe. To permit exposure of the ventral surface of the brain, anastomotic veins were coagulated and the occipitotem- poral convexity was retracted gently. 'The cortex and underlying white matter just medial to the occipitotemporal sulcus were removed by suction. The exposed hippocampus then was aspirated with a 19 gauge sucker, with the roof of the lateral ventricle used as a guide.

\section{Histology}

After fixation of the brains in sugar/formalin, frozen sections were cut at $25-\mu \mathrm{m}$ thickness and alternate sections were stained with cresyl violet for cellular Nissl substance or with the Weil hematoxylin method for fibers. In all five monkeys in the AIT' group, both the lesions and the subsequent pattern of retrograde degeneration in the pulvinar corresponded closely to the removals and thalamic degeneration seen in monkeys in group AIT in experiment 1 (Fig. 2).

All seven monkeys in group HT had complete bilateral and symmetrical removals of hippocampus (Fig. 3). In addition, each animal sustained bilateral damage to cortical areas TF-TH-minimally, in two cases (HT-1 and HT-2), moderately, in two (HT-3 and HT-4), and extensively in three others (HT-5, 6, and 7). However, in none of the monkeys was there direct damage to either the anterior inferotemporal or entorhinal cortex or the amygdala. The temporal stem was also spared, except for monkey HT-4 which had unilateral damage to the stem in the right hemisphere.

\section{Apparatus and Procedure}

\section{Visual tasks}

The apparatus and behavioral procedures were the same as those described in experiment 1 . The animals first were pretrained to displace a gray plaque for food reward and then were given three individual visual discriminations, i.e., brightness, hue, and pattern. Following this, they were taught concurrent object discrimination tasks I and II.

\section{Tactual tasks}

Upon completion of the visual task, animals were trained to work in the dark, tested on a series of five individual tactual discriminations and, finally, tested on the tactual concurrent task. Testing was conducted in a modified Wisconsin General Testing Apparatus. Two infrared light sources, powered by an EICO 10648 power supply, were fixed to the ceiling of the testing chamber. A binocular infrared light detector allowed continuous observation of on-going behavior in complete darkness. Illumination levels in the chamber were controlled by a rheostat. Two boxes measuring $11 \times 6 \times 5 \mathrm{~cm}$ each were mounted $30 \mathrm{~cm}$ apart, center to center, on a testing board. Test objects were placed over the boxes on movable lids so that as soon as the screen was raised, they could be easily reached and displaced, exposing a food well centered in each box.

\section{Pretraining: Gradual transition from visual to tactual discrimination}

Monkeys were trained first under normal illumination with one pair of objects (wooden sphere versus plastic junk object) mounted on wooden bases. During the first three daily sessions, consisting of 30 trials each, illumi- 

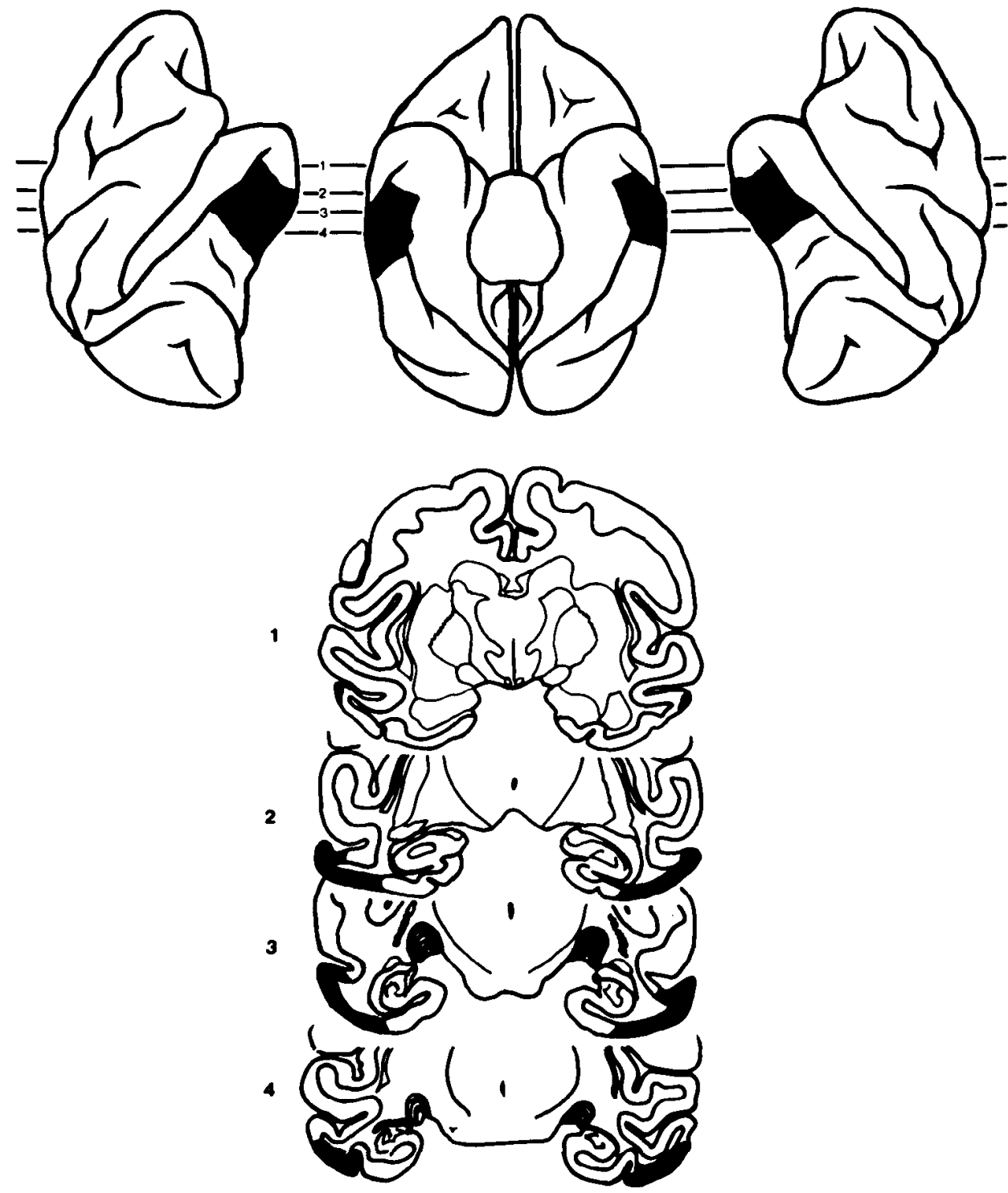

AIT

Figure 2. Composite tracings of the lateral and ventral aspects of the brain and representative coronal sections showing the typical lesion in anterior inferotemporal group AIT' (experiment 2).

nation was gradually lowered, within and between sessions, until the monkeys were able to work in complete darkness. Training was continued until a learning criterion of 90 correct responses in 100 consecutive trials was reached in the dark.

Individual discriminations. Five pairs of objects, each differing along one or more dimensions (shape, size, and texture) were presented in the following order: pair 1, small wooden ball versus plastic bottle; pair 2, plastic animal versus plastic cup; pair 3, large cork versus small cork; pair 4, small wooden cube versus wire mesh cube; pair 5 , wooden pyramid versus wooden sphere. The first member of each pair was positive and the locations of the two objects changed from left to right in a predetermined order (Gellerman, 1933). As in the case of individ- ual visual tasks, each pair of objects was taught separately using a non-correction method. Thirty trials a day were given, 6 days a week, until a learning criterion of 90 correct in 100 consecutive trials was reached.

Concurrent discrimination task. This task was administered following the same procedure as that used for visual concurrent task I. Eight new pairs of objects, each differing along one or more dimensions, were presented in an intermingled fashion in each testing session so that all eight discriminations had to be learned simultaneously. Forty trials a day were given, 6 days a week, until a learning criterion of 39 correct responses in 40 consecutive trials was met in one session. Unlike in the visual modality, however, monkeys were not trained on concurrent discrimination II (stages a to $\mathrm{d}$ ). 

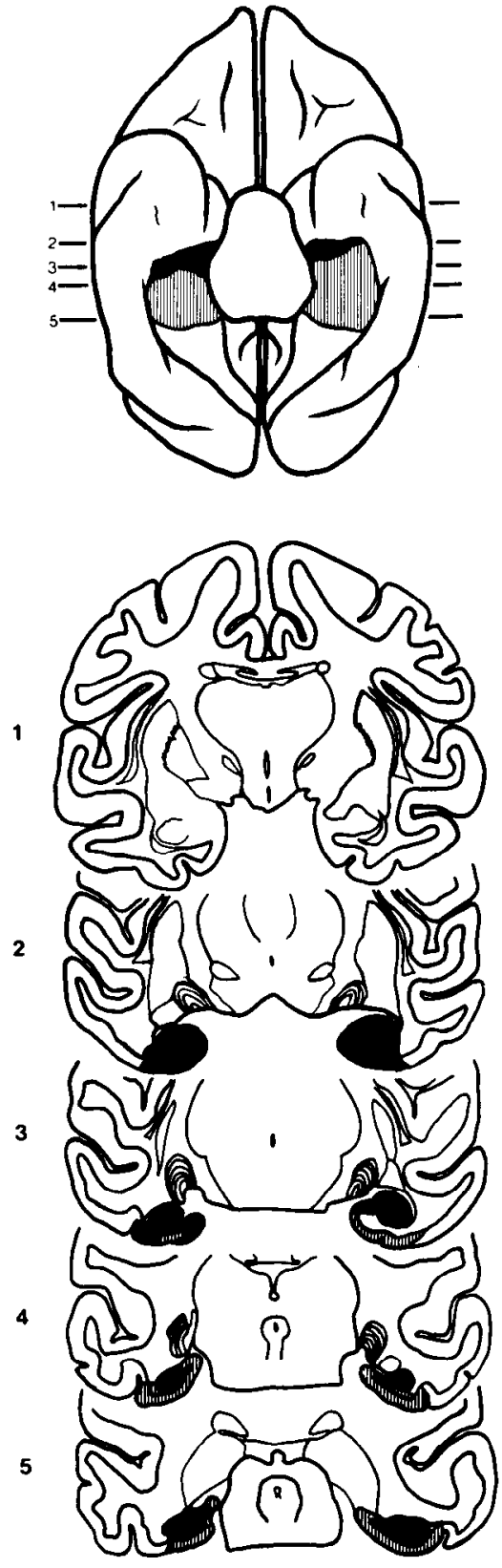

HT

Figure 3. Composite tracings of the ventral aspect of the brain and representative coronal sections showing the smallest and largest lesions in hippocampal group HT (experiment 2). Solid black represents damage common to all cases; striped areas indicate extent of largest lesion.

\section{Results}

\section{Visual Tasks}

\section{Individual discriminations}

The results obtained on brightness and hue discrimination tasks are summarized in the lower portion of Table I. Overall group comparisons yielded no significant differences in performance on either task, though individual animals in groups $\mathrm{AIT}^{\prime}$ and $\mathrm{HT}$ obtained elevated error scores. 'The data obtained with pattern discrimination are shown in Table IV. An overall group comparison yielded an $H$ value of 7.17 , significant at better than the 0.05 level of confidence. Both groups of operated monkeys, not significantly different from each other, were significantly impaired compared to the normal control group $(U=0 ; p=0.016$ and $U=3 ; p=0.042$, for $\mathrm{AIT}^{\prime}$ and HT groups, respectively).

Though the amount of hippocampal removal in each of the seven HT monkeys was comparable, there were differences among animals in the amount of additional damage to area TF-TH resulting in appreciable individual differences in the total size of lesion. However, the Spearman rank correlation between total size of lesion and error scores on the visual pattern task failed to reveal a significant relationship $\left(r_{s}=0.57 ; p>0.05\right)$.

\section{Concurrent discrimination I}

The results are shown in Table IV. An overall group comparison yielded a statistically significant $H$ value of $9.83(p<0.01)$. Monkeys in either $\mathrm{AIT}^{\prime}$ or HT groups made significantly more errors than did those in the normal, control group $(U=0 ; p=0.016$ and $U=0 ; p=$ 0.006 , respectively, for the two groups), with no significant differences between the two operated groups. For group HT, no significant correlations were found either between size of lesion and errors on the concurrent task $\left(r_{s}=0.11 ; p>0.05\right)$ or between errors on the pattern discrimination and the concurrent task $\left(r_{s}=0\right)$.

TABLE IV

Experiment 2: Visual modality

These data show the effects of inferotemporal and hippocampal ablations on visual pattern and concurrent discrimination tasks. The symbol, + , denotes failure to reach learning criterion within limits of testing. Animals in the hippocampal group are coded with respect to size of total lesion (smallest, 1), with low variability in size of the lesion in group AIT'. Animals in each group are ordered with respect to errors in the concurrent task.

\begin{tabular}{ccccrr}
\hline & \multicolumn{2}{c}{ Pattern } & \multicolumn{2}{c}{ Concurrent I } \\
\cline { 2 - 3 } \cline { 5 - 6 } Group & Errors & $\begin{array}{c}\text { \% Correct of } \\
\text { Last 100 Trials }\end{array}$ & Trials & Errors \\
\hline Normal & & & & \\
$N^{\prime}-1$ & 231 & 90 & 240 & 81 \\
$N^{\prime}-2$ & 101 & 90 & 320 & 105 \\
$N^{\prime}-3$ & 203 & 91 & 440 & 114 \\
$N^{\prime}-4$ & 207 & 90 & 480 & 120
\end{tabular}

\section{Anterior}

inferotemporal

AIT'-1 $^{\prime}$

AIT' $^{\prime}-2$

AIT'-3

AIT'-4

AIT $^{\prime}-5$

$$
\begin{aligned}
& 398+ \\
& 406+ \\
& 254+ \\
& 404+ \\
& 397+
\end{aligned}
$$

48
50
69
49
49

$\begin{array}{rr}840 & 221 \\ 1080 & 301 \\ 1040 & 324 \\ 920 & 341 \\ 1200 & 382\end{array}$

Hippocampal

HT-2

HT-7

142

HT-5

$379+$

HT-4

$413+$

HT-1

$403+$

HT-6

258

HT-3

364

$\begin{array}{ll}640 & 159 \\ 560 & 191 \\ 960 & 242 \\ 740 & 252 \\ 680 & 255 \\ 200 & 270 \\ 040 & 373\end{array}$


Note: Group AIT' made significantly more errors than did the more experimentally sophisticated group AIT in experiment $1(U=3 ; p=0.056)$.

\section{Concurrent discrimination II}

Stage $a$. There were no significant differences among groups in the number of errors on the five problems. Mean errors were 30.0, 34.8, and 32.3 for the normal control, $\mathrm{AIT}^{\prime}$, and $\mathrm{HT}$ groups, respectively.

Stage $b$. All groups retained the five discriminations learned in stage a. Mean error scores were 2.0, 2.0, and 2.9 for the three groups, respectively.

Stage $c$. There were no significant differences in the number of errors to learning criterion among groups when the five object pairs were presented concurrently. Mean error scores ranged between 3.1 and 3.9 for all groups.

Stage $d$. When five new pairs of objects were presented concurrently with the five familiar pairs, overall group differences in learning scores were significant $(H=9.10$; $p<0.02$ ). Frrors obtained by the normal, control group ranged between 25 and 43 , with a mean of 35.3. Monkeys in $\mathrm{AIT}^{\prime}(\bar{X}=82.2)$ and HT $(\bar{X}=66.7)$ groups made significantly more errors than did those in the normal group ( $U$ values $=0 ; p=0.016$ and $<0.006$, respectively, for the two groups) but did not differ significantly from each other.

There were no significant differences among groups in the number of errors made with the five familiar pairs of objects, with group mean errors ranging from $3.7\left(\mathrm{~N}^{\prime}\right)$ to $6.4(\mathrm{HT})$. As in experiment 1, monkeys in each group accumulated most of their errors on the five new, as opposed to the five familiar, pairs of objects. Thus, while the mean number of errors made by the three groups with the familiar five pairs ranged from 7.2 (AIT') to 12.5 $\left(\mathrm{N}^{\prime}\right)$, those made with the five new pairs ranged from 87.5 $\left(\mathrm{N}^{\prime}\right)$ to $92.5\left(\mathrm{AIT}^{\prime}\right)$.

\section{Tactual Tasks}

Data will be described for 14 of the 16 monkeys trained previously in the visual modality; monkeys $\mathrm{AIT}^{\prime}-5$ and HT-5 refused to work in total darkness and had to be dropped from the study.

\section{Individual discriminations}

No significant group differences were found on the five pretraining tasks. Group mean errors to learning criterion for all five problems were 260.8 (range: 136 to 430), 306.5 (range: 182 to 412 ), and 317.7 (range: 97 to 492 ) for $\mathrm{N}^{\prime}$, AIT', and HT groups, respectively.

\section{Concurrent discrimination I}

Results are shown in Table V. An overall group comparison yielded an $H$ value of 6.56 , significant at better than the 0.05 level of confidence. Due to the efficient performance of two of the six monkeys in group HT (HT3 and HT-7), this group did not differ significantly from the normal group. However, group HT did make significantly more errors than did group $\mathrm{AIT}^{\prime}(U=0.5 ; p<$ 0.02 ), whose learning scores were well within the normal range.
TABLE V

Experiment 2: Tactual modality

These data show the effects of inferotemporal cortical and hippocampal lesions on concurrent discrimination task I. Animals in the hippocampal group are coded with respect to size of lesion (smallest, 1), with low variability in the size of the lesion in group AIT'. Animals in each group are ordered with respect to increasing number of errors.

\begin{tabular}{lcc}
\hline \multicolumn{1}{c}{ Group } & Trials & Errors \\
\hline Normal & & \\
$N^{\prime}-1$ & 400 & 111 \\
$N^{\prime}-2$ & 560 & 146 \\
$N^{\prime}-3$ & 760 & 171 \\
$N^{\prime}-4$ & 960 & 192 \\
& & \\
Anterior inferotemporal & & \\
AIT'-2 & 400 & 135 \\
AIT'-3 & 440 & 150 \\
AIT'-1 & 600 & 152 \\
AIT'-4 & 720 & 161 \\
& & \\
Hippocampal & & \\
HT-3 & 880 & 161 \\
HT-7 & 640 & 162 \\
HT-6 & 1040 & 243 \\
HT-1 & 1020 & 248 \\
HT-2 & 1200 & 269 \\
HT-4 & 1200 & 319 \\
\hline
\end{tabular}

\section{Performance Patterns}

As in experiment 1, we tabulated, for each monkey, the number of errors made with each of the eight pairs of objects, day by day, throughout all testing sessions until learning criterion was reached on the task. We said that a given pair was learned when, on 2 consecutive days, no more than one error was made with that pair. We found that normal, control monkeys were able to learn several individual discriminations simultaneously (clustered pattern) and that, once learned, these discriminations were well retained on subsequent sessions. This pattern was found in both visual and tactual modalities. In contrast, all monkeys in group HT showed only a distributed pattern, with long intervening pauses between acquisition of individual pairs in the visual modality. The same was true of the four HT monkeys that were impaired in the tactual mode. Group AIT' showed only a distributed pattern when impaired (in the visual mode) but only a clustered pattern when not impaired (tactual mode).

When we examined the point at which operated monkeys began to experience more difficulty than did normal monkeys in learning successive pairs, the following patterns of performance emerged.

\section{Visual modality}

Monkeys in group AIT' $^{\prime}$ began to have significantly more difficulty than normal monkeys as early as with the second pair (Fig. 4). This difference in performance between the two groups remained significant through the remaining six pairs ( $U$ values $=0 ; p$ values $=0.016$ ) Group HT handled the first three pairs relatively efficiently, but took significantly more sessions than did normal monkeys to learn each of the remaining five pairs $(U$ values $=0 ; p$ values $=0.006)$. 


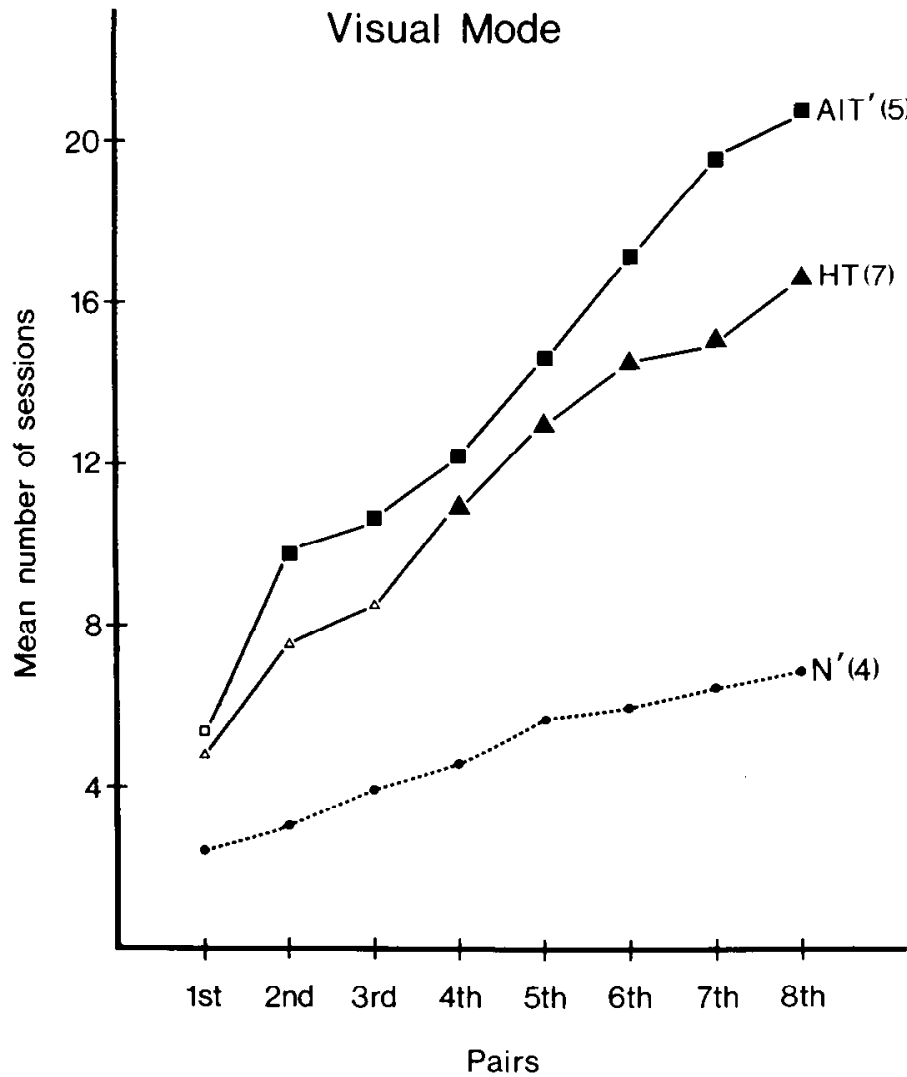

Figure 4. Experiment 2, visual modality: Rates at which three groups of monkeys learned successively each of the eight discrimination problems presented concurrently. Larger solid symbols indicate significant differences from the normal, control group.

The greater difficulty experienced by monkeys in the two operated groups appeared much earlier than it did in experiment 1 , when significantly greater than normal difficulty was noted only with the sixth, seventh, and eighth pairs.

\section{Tactual modality}

The slopes of the curves reflecting the rate of learning of eight successive pairs were comparable for normal control monkeys and those in group AIT' (Fig. 5). However, the four impaired monkeys in group HT needed significantly more sessions than did normal monkeys to learn their fourth, seventh, and eighth pairs ( $U$ values $=1 ; p$ values $=0.058$, for the three comparisons). Significant differences between the four impaired HT monkeys and those in group AIT' were noted on the fourth $(U=1 ; p=0.058)$ and eighth $(U=2 ; p=0.057$, onetailed test) pairs.

\section{Discussion}

The main findings were: (1) Selective ablations of the hippocampal formation or those of entorhinal area may produce as severe a deficit in performance on visual concurrent discrimination tasks as do ablations of the anterior inferotemporal cortex (Fig. 6). (2) The deficit after hippocampal resections may be independent of sensory modality, while that which follows inferotem- poral ablations is specific to the visual mode. (3) Fornix transections had no deleterious effects. The latter finding will be discussed first.

Sections of the fornix. In contrast with other operated groups, monkeys with transections of the fornix system were not impaired on concurrent discrimination tasks I and II. Given previous practice with other tasks, recovery of function cannot be confidently ruled out. Yet, monkeys with circumscribed entorhinal lesions that shared the same experimental history remained impaired. More convincing, perhaps, is the fact that our findings are in accord with those of Gaffan (1974) who reported the absence of immediate postoperative impairment after fornix sections on a matching to sample task. As in the case of the concurrent discrimination task, monkeys in Gaffan's study were required to form a series of correct associations between objects and their reward value.

Current anatomical evidence provides a basis for possible functional dissociation of effect between fornix sections and entorhinal ablations. While both types of lesion de-afferent and de-efferent the hippocampal formation, the structures from which they disconnect it differ. The fornix-fimbria system in the monkey relays a large number of fibers which, together, connect reciprocally the hippocampus with several areas in the septal complex and hypothalamus (Valenstein and Nauta, 1959; Poletti and Creswell, 1977; Krayniak et al., 1979; Swanson and Cowan, 1979; Wyss et al., 1979; Amaral and Cowan, 1980; DeVito, 1980). In contrast, caudally directed efferents of

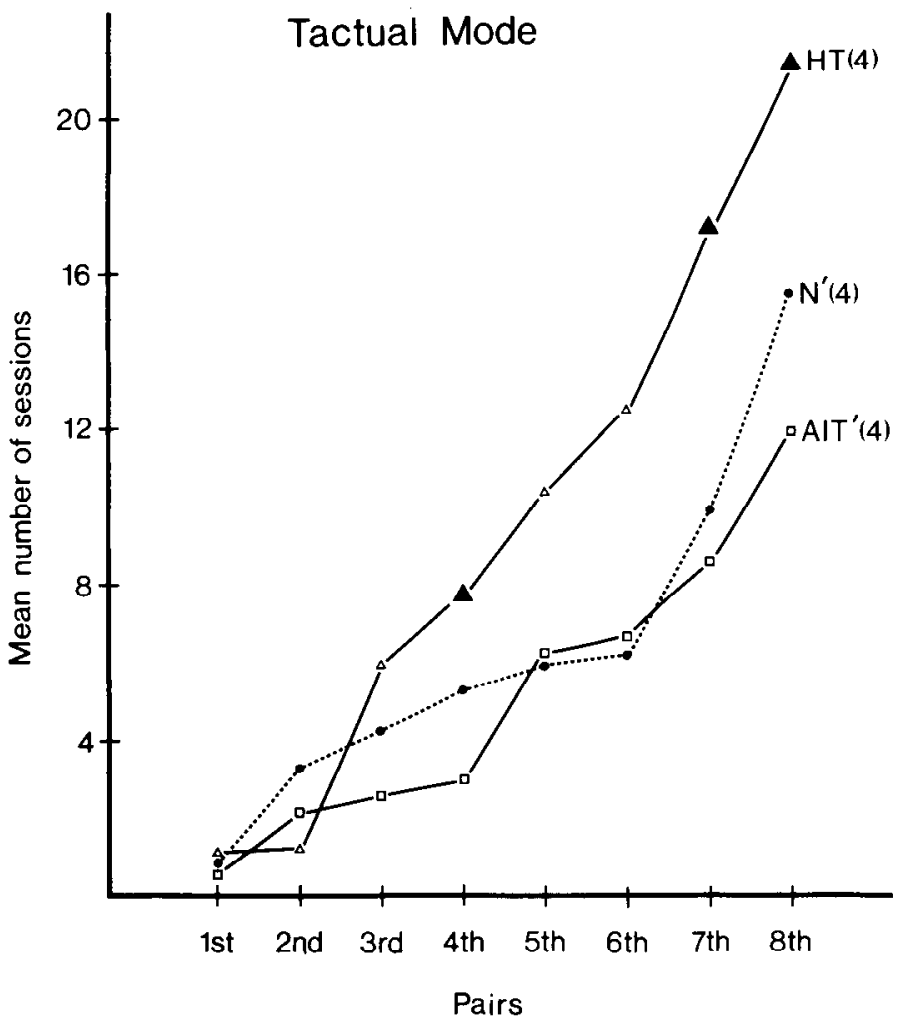

Figure 5. Experiment 2, tactual modality: Rates at which three groups of monkeys learned successively each of the eight discrimination problems presented concurrently. Larger solid symbols indicate significant differences from the normal, control group. 


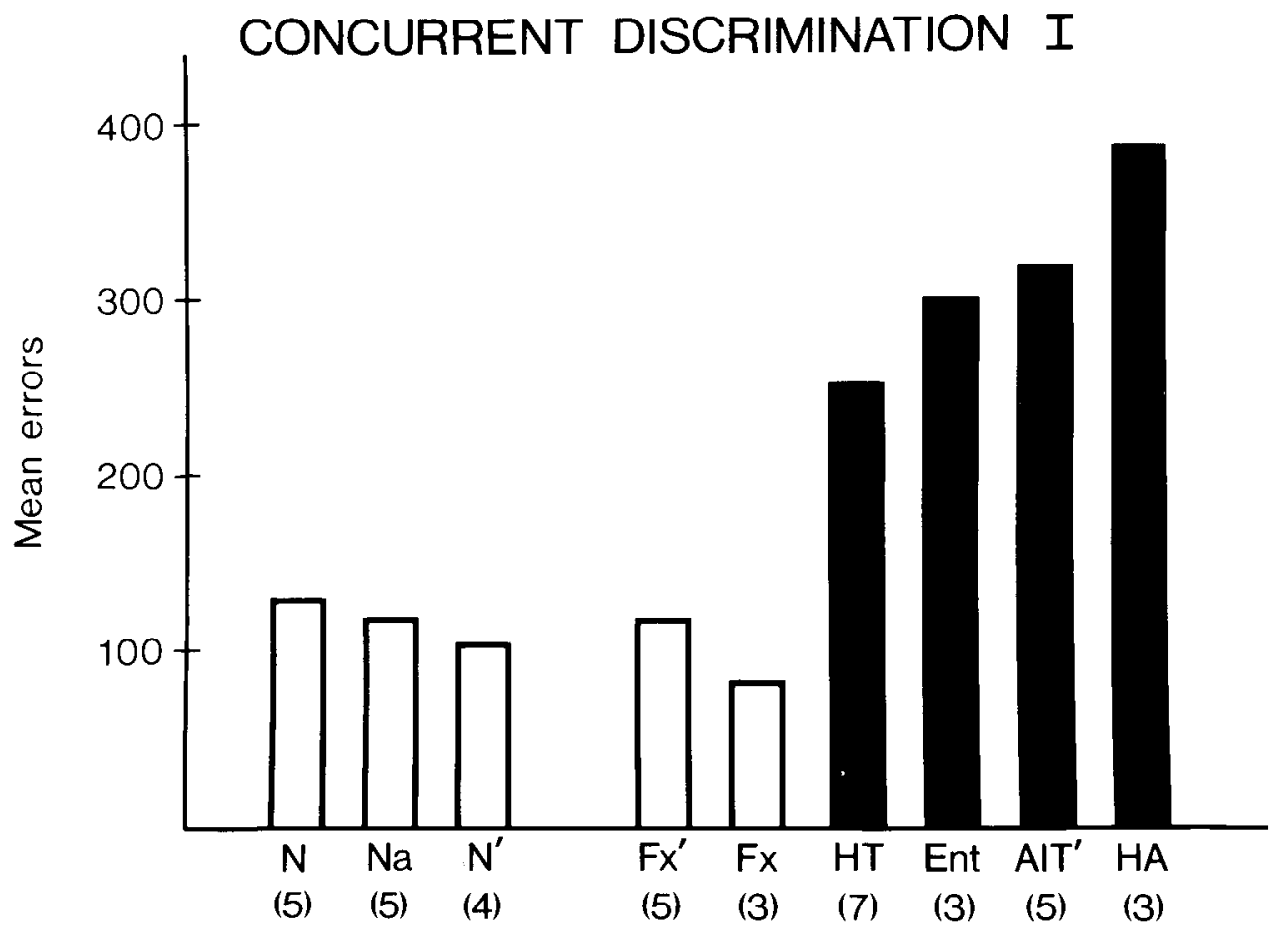

Figure 6. Experiments 1, 1a, and 2: Performance on the concurrent discrimination task in the visual modality of normal, control, and operated monkeys. Solid bars indicate significant impairment in relation to respective normal control groups. $N$, normal (experiment 1); $N a$, normal (experiment 1a); $N^{\prime}$, normal (experiment 2); $F x^{\prime}$, fornix (experiment 1a); $F x$, fornix (experiment 1); $H T$, hippocampal (experiment 2); Ent, entorhinal (experiment 1); $A I T^{v}$, anterior inferotemporal (experiment 2); $H A$, hippocampal with additional inferotemporal damage (experiment 1). Numbers in parentheses represent the number of monkeys in each group.

the hippocampus project through the subicular complex to terminate in the rhinal cortical areas (Rosene and Van Hoesen, 1977). Thus, transection of the fornix leaves this caudal output intact, as it also spares massive afferent pathways to the hippocampus through areas TF-TH and entorhinal cortex. Our data strongly suggest that the integrity of this caudal reciprocal projection system is sufficient to allow unimpaired learning of concurrent object discriminations. The finding that a lesion of the entorhinal area or that of area TF-TH is sufficient to disrupt this type of learning supports the notion.

An additional anatomical point is pertinent. While two of the three monkeys with fornix sections performed better than did normal monkeys, monkey Fx-3 (experiment 1), with additional damage of the hippocampal commissure, obtained a much higher learning score (Table II). The same monkey was also found to be impaired in retention of object-reward associations in a previous study (Mahut et al., 1981). This is of interest in light of recent clinical data; for the most part, fornix damage in patients does not produce memory deficits (for reviews, see Woolsey and Nelson, 1975; Squire and Moore, 1979). However, an amnesic syndrome has been observed in a case with posterior fornix damage in which a long-standing neoplasm encroached on the hippocampal commissure (Heilman and Sypert, 1977).

Anterior inferotemporal ablations. In accord with earlier studies (Iwai and Mishkin, 1968; Cowey and Gross, 1970 ), monkeys with inferotemporal removals were impaired in visual concurrent discrimination learning and a detailed analysis of performance revealed that they were susceptible to both pro- and retroactive interpair interference (Fig. 4). The deficit was accentuated in unsophisticated animals (group $\mathrm{AIT}^{\prime}$, experiment 2), but became attenuated as a result of extensive prior visual discrimination training (group AIT, experiment 1). Yet, monkeys with hippocampal-inferotemporal ablations showed no evidence of the beneficial influence of practice (group HA, experiment 1). In fact, two of the three HA monkeys were the only operated animals not to have reached learning criterion within the limits of testing. The greater severity of the deficit after combined lesions extends the results of a study by Iversen (1970) in which baboons with inferotemporal-hippocampal ablations were more severely impaired on a visual discrimination retention task than were those with inferotemporal removals alone. Mass action effects, in both Iversen's study and ours, cannot be dismissed. Nevertheless, it is conceivable that additional hippocampal damage may exacerbate the deleterious effects of an inferotemporal lesion. Such a notion is compatible with clinical findings which reveal that the inclusion of hippocampus in a unilateral temporal lobe resection accentuates the typical hemisphere-specific postoperative defects (Milner, 1974).

The efficient acquisition of concurrent discrimination tasks in the tactual modality by monkeys in group AIT' (experiment 2) was not surprising since inferotemporal lesions are known to impair performance on visual, and only visual, tasks (Wilson, 1957; Iversen, 1967).

Hippocampal resections. Monkeys with removals of hippocampus or entorhinal area not impaired in learning individual discriminations were markedly impaired in 
learning several discriminations simultaneously. A more detailed, day by day, analysis of their performance suggests that they are more susceptible to pro- and retroactive interpair interference than are normal, control monkeys and, in this respect, their performance paralleled that of monkeys with inferotemporal cortical removals. Interestingly, the point at which the effects of interference began to affect performance of operated animals depended, in part, on their history; groups of experimentally sophisticated monkeys with hippocampal removals learned as many as five discriminations at a relatively normal rate. However, less experienced monkeys could not handle more than one to three pairs with normal ease.

Our findings extend the results of two earlier studies in which learning deficits of monkeys with temporal lobe ablations that included the hippocampus were attributable more to interference than to an abnormally rapid decay of memory traces. Thus, Iversen (1970) found that baboons with inferotemporal-hippocampal removals were not impaired in retaining simple visual object discriminations when 15 min of darkness or performance of a previously learned tactile discrimination separated acquisition from retention tests. Similarly, Correll and Scoville (1970) found that the impairment on a serial visual learning task by monkeys with amygdalo-hippocampal resections could be significantly reduced when the intertrial interval was increased from 10 to $45 \mathrm{sec}$, presumably attenuating interpair interference. More recently, on a nonmatching to sample task, similar to that described by Mishkin (1978), monkeys with hippocampal resections were severely impaired when the number of objects presented consecutively for familiarization was increased from 1 to 10 (lists of $1,3,5$, and 10 objects) and the degree of impairment was related to the length of lists (Rehbein et al., 1980; Moss et al., 1980). Presumably, longer lists provided both retro- and proactive interference. It should be noted that, on another version of the same recognition task, when instead of lists, delays were interposed between the presentation of sample and test objects, monkeys with hippocampal resections still obtained elevated error scores, but the degree of impairment was less severe than on the list version (Rehbein et al., 1980; Moss et al., 1980).

Though monkeys with resections of hippocampus appear to be particularly sensitive to interference from competing items, they also show deficits on simple twochoice discrimination tasks. Thus, they were impaired on a task in which they had to remember whether choices of one of two equally familiar objects had been rewarded, or not rewarded, the day before and this impairment in learning object-reward associations was present in both visual and tactual modalities (Task $A-B$, Mahut et al., 1979,1981 ). Similarly, in the present study, individual operated monkeys obtained elevated error scores on brightness and hue discriminations and were significantly impaired, as a group, on the pattern discrimination task (Table IV), even though none had sustained additional damage of the inferotemporal cortex (group HT, experiment 2).

Taken together, the existing evidence reveals a deficit on tasks which require not only object recognition, but also the capacity to form and retain object-reward associations in more than one modality.

Supramodal effects of hippocampal resections. Both hippocampal and inferotemporal ablations were followed by impairment on the concurrent task in the visual modality. Yet, on a task which required object-reward associations described above (Task $A-B$, Mahut et al., 1979,1981 ), only monkeys with hippocampal, but not those with inferotemporal, ablations were impaired. Furthermore, other authors report that monkeys with inferotemporal ablations are more severely impaired in object recognition (Mishkin and Oubre, 1976) than they are in learning to associate objects with their reward value (Spiegler and Mishkin, 1978). Conceivably, therefore, monkeys with inferotemporal ablations were impaired in the present study because of a primary defect in recognizing the physical characteristics of several pairs of objects presented concurrently in the visual modality, whereas the impairment of monkeys with hippocampal resections may have been due, primarily, to a deficit in retention of object-reward associations, which was independent of modality.

More direct evidence, however, is needed to determine to what extent, if any, the two structures function independently or together. The unexpected finding of impairment on a spatial reversal task after inferotemporal ablations (M. Moss, unpublished data), a defect which mirrors the typical consequence of hippocampal resections in the visual (Mahut, 1971) and tactual (Mahut and Zola, 1973) modes, hints at the possibility that, at least to some extent, certain effects of inferotemporal ablations may be attributed to a disruption of normal hippocampal functions.

The possible supramodal involvement of the hippocampus in associative learning and memory raises an important anatomical question: If the hippocampus were to serve such a function, what are the afferent inputs which provide both visual and tactual information? In the case of visual input, it is known that the hippocampus is linked to the primary visual cortex by a sequence of cortical projections through the prestriate and inferotemporal areas (Kuypers et al., 1965; Pandya and Kuypers, 1969; Jones and Powell, 1970) which are relayed, in turn, to both entorhinal and parahippocampal areas (Whitlock and Nauta, 1956; Jones and Powell, 1970; Moss, 1974; Seltzer and Pandya, 1974; Van Hoesen and Pandya, 1975a) and, thence, to the hippocampus (Van Hoesen and Pandya, 1975b; Van Hoesen, 1980).

In the case of tactual input, connections between the primary somatosensory cortices and the limbic system, through the inferior parietal areas, are only now investigated in detail (Vogt and Pandya, 1978; Pandya et al., 1979; Seltzer and Van Hoesen, 1979; Van Hoesen, 1980; Seltzer and Pandya, 1980). Unlike the sequential neural systems in vision, the functional contribution of the tactual pathways remains unclear. In fact, recent behavioral and single-unit studies suggest that the inferior parietal lobule, the pivotal link in these connections, may be more concerned with visuospatial than with tactual functions (see Mishkin, 1979). Yet, as suggested by Mish- 
kin, there may be a functional link between the primary somatosensory cortices and the limbic system through the secondary somatosensory cortex (SII) and posterior insula, with area SII representing a tactual analogue to the inferotemporal areas in vision. Preliminary evidence of a connection between the posterior insula and entorhinal cortex (M. -M. Mesulam and E. Mufson, personal communication) provides an important anatomical link in a potential somatolimbic relay. Thus, entorhinal cortex stands as a convergence site of widespread connections from cortical association areas concerned with the processing of visual and tactual information. The hippocampus, by virtue of its massive connections with rhinal cortices, is the recipient of such multisensory input and may, therefore, be essential for mediating associative learning and memory in more than one modality.

\section{Comment}

A question related to the effects of hippocampal resections is raised by the role attributed recently to the temporal stem. After a careful review of clinical and experimental material, Horel (1978) suggested that it is not damage to the hippocampus but rather that of the adjacent temporal stem which is responsible for memory and learning deficits reported by several authors. Since a portion of the stem consists of fibers with cells of origin in the inferotemporal cortex (Whitlock and Nauta, 1956), it was nol surprising to see degeneration in the temporal stem of our monkeys with hippocampal removals that had additional damage of the inferotemporal cortex (group HA, experiment 1). However, it should be emphasized that, in experiment 2, monkeys with hippocampal resections but no inferotemporal damage and no direct damage to the temporal stem (group HT) were still significantly impaired on the visual concurrent task. In view of this negative evidence, the role of the temporal stem in memory remains an open question.

\section{References}

Amaral, D. G., and W. M. Cowan (1980) Subcortical afferents to the hippocampal formation in the monkey. J. Comp. Neurol. 189: 573-591.

Correll, R. E., and W. B. Scoville (1965) Effects of medial temporal lesions on visual discrimination performance. J. Comp. Physiol. Psychol. 60: 175-181.

Correll, R. E., and W. B. Scoville (1970) Relationship of ITI to acquisition of serial visual discriminations following temporal rhinencephalic resection in monkeys. J. Comp. Physiol. Psychol. 3: 464-469.

Cowey, A., and C. G. Gross (1970) Effects of foveal prestriate and inferotemporal lesions on visual discrimination by rhesus monkeys. Exp. Brain Res. 11: 128-144.

Dean, P. (1976) Effects of inferotemporal lesions on the behavior of monkeys. Psychol. Bull. 83: 41-71.

DeVito, J. L. (1980) Subcortical projections to the hippocampal formation in squirrel monkey (Saimiri sciureus). Brain Res. Bull. 3: 285-289.

Gaffan, D. (1974) Recognition impaired and association intact in the memory of monkeys after transection of the fornix. $J$. Comp. Physiol. Psychol. 86: 1100-1109.

Gellerman, L. W. (1933) Chance orders of alternating stimuli in visual discrimination experiments. J. Gen. Psychol. 42: 207208.

Gross, C. G. (1973) Visual functions of inferotemporal cortex. In Handbook of Sensory Physiology, R. Jung, ed., pp. 451482, Springer-Verlag, Berlin.

Heilman, K. M., and G. W. Sypert (1977) Korsakoff's syndrome resulting from bilateral fornix lesions. Neurology (N. Y.) 27: 490-493.
Horel, J. (1978) The neuroanatomy of amnesia. A critique of the hippocampal memory hypothesis. Brain 101: 403-445.

Iversen, S. D. (1967) Tactile learning and memory in baboons after temporal and frontal lesions. Exp. Neurol. 18: 228-238.

Iversen, S. D. (1970) Interference and inferotemporal memory deficits. Brain Res. 19: 277-289.

Iversen, S. D. (1976) Do hippocampal lesions produce amnesia in animals? In International Review of Neurobiology, C. C Pfeiffer and J. R. Smythies, eds., pp. 1-49, Academic Press, New York.

Iwai, E., and M. Mishkin (1968) Two visual foci in the temporal lobe of monkeys. Japan-US Joint Seminar on Neurophysi. ological Basis of Learning and Behavior, Kyoto, Japan.

Jones, E. G., and T. P. S. Powell (1970) An anatomical study of converging sensory pathways within the cerebral cortex of the monkey. Brain 93: 783-820.

Krayniak, P. F., A. Siegel, R. C. Meibach, D. Fruchtman, and M. Scrimenti (1979) Origin of the fornix system in the squirrel monkey. Brain Res. 160: 401-411.

Kuypers, H. G. J. M., M. K. Szwarcbart, M. Mishkin, and H. E. Rosvold (1965) Occipitotemporal cortico-cortical connections in the rhesus monkey. Exp. Neurol. 11: 245-262.

Mahut, H. (1971) Spatial and object reversal learning in monkeys with partial temporal lobe ablations. Neuropsychologia 9: 409-424.

Mahut, H., and S. Zola (1973) A non-modality specific impairment in spatial learning after fornix lesions in monkeys. Neuropsychologia 11: 255-269.

Mahut, H., S. Zola-Morgan, and M. Moss (1979) Retention deficits in monkeys after damage to the hippocampal system. The INS Bulletin, December, p. 12.

Mahut, H., M. Moss, and S. Zola-Morgan (1981) Retention deficits after combined amygdalo-hippocampal and selective hippocampal resections in the monkey. Neuropsychologia 19: in press.

Milner, B. (1974) Hemispheric specialization: Scope and limits. In The Neurosciences, Third Study Program, F. O. Schmitt and F. G. Worden, eds., pp. 75-89, M. I. T. Press, Cambridge.

Mishkin, M. (1978) Memory in monkeys severely impaired by combined but not separate removal of amygdala and hippocampus. Nature 273: 297-298.

Mishkin, M. (1979) Analogous neural models for tactual and visual learning. Neuropsychologia 17: 139-151.

Mishkin, M., and J. L. Oubre (1976) Dissociation of deficits on visual memory tasks after inferior temporal and amygdala lesions in monkeys. Soc. Neurosci. Abstr. 2: 1127.

Moss, M. (1974) Differential projections of two sectors of the inferotemporal cortex in the rhesus monkey. Soc. Neurosci. Abstr. 346.

Moss, M., H. Mahut, and S. Zola-Morgan (1980) Associative and recognition impairments in monkeys after hippocampal resections. Soc. Neurosci. Abstr. 6: 192.

Pandya, D. N., and H. G. J. M. Kuypers (1969) Cortico-cortical connections in the rhesus monkey. Brain Res. 13: 13-36.

Pandya, D. N., G. W. Van Hoesen, and M.- M. Mesulam (1979) The cortical projections of the cingulate gyrus in the rhesus monkey. Anat. Rec. 193: 643-644.

Poletti, C. E., and G. Creswell (1977) Fornix system efferent projections in the squirrel monkey: An experimental degeneration study. J. Comp. Neurol. 175: 101-128.

Rehbein, L., S. Zola-Morgan, H. Mahut, and M. Moss (1980) Failure of sparing, or recovery, of recognition memory after early hippocampal resections in the rhesus macaque. Soc. Neurosci. Abstr. 6: 88.

Rosene, D. L., and G. W. Van Hoesen (1977) Hippocampal efferents reach widespread areas of cerebral cortex and amygdala in the rhesus monkey. Science 198: 315-317. 
Seltzer, B., and D. N. Pandya (1974) Some cortical projections to the parahippocampal area of the rhesus monkey. Exp. Neurol. 34: 212-225.

Seltzer, B., and D. N. Pandya (1980) Converging visual and somatic sensory cortical input to the intraparietal sulcus of the rhesus monkey. Brain Res. 192: 339-351.

Seltzer, B., and G. W. Van Hoesen (1979) A direct inferior parietal lobule projection to the presubiculum in the rhesus monkey. Brain Res. 179: 157-161.

Spiegler, B. J., and M. Mishkin (1978) Evidence for the sequential participation of inferior temporal cortex and amygdala in stimulus-reward learning. Soc. Neurosci. Abstr. 4: 263.

Squire, I. R., and R. Y. Moore (1979) Dorsal thalamic lesion in a noted case of human memory dysfunction. Ann. Neurol. 6: 503-506.

Swanson, L. W., and W. M. Cowan (1979) The connections of the septal region in the rat. J. Comp. Neurol. 186: 621-656.

Valenstein, E. S., and W. J. H. Nauta (1959) A comparison of the distribution of the fornix system in the rat, guinea pig, cat and monkey. J. Comp. Neurol. 3: 337-363.

Van Hoesen, G. W. (1980) The cortico-cortical projections of the posterior parahippocampal area in the rhesus monkey. Anat. Rec. 196: 195A.

Van Hoesen, G. W., and D. N. Pandya (1975a) Some connec- tions of the entorhinal (area 28) and perirhinal (area 35) cortices of the rhesus monkey. I. Temporal lobe afferents. Brain Res. 95: 1-24.

Van Hoesen, G. W., and D. N. Pandya (1975b) Some connections of the entorhinal (area 28) and perirhinal (area 35) cortices of the rhesus monkey. III. Efferent connections. Brain Res. 95: 48-67.

Vogt, B. A., and D. N. Pandya (1978) Cortico-cortical connections of somatic sensory cortex (areas 3,1 and 2) in the rhesus monkey. J. Comp. Neurol. 177: 179-192.

von Bonin, G., and P. Bailey (1947) The Neocortex of Macaca mulatta, University of Illinois Press, Urbana.

Whitlock, D. G., and W. J. H. Nauta (1956) Subcortical projections from the temporal neocortex in Macaca mulatta. J. Comp. Neurol. 106: 183-212.

Wilson, M. (1957) Effects of circumscribed cortical lesions upon somesthetic and visual discrimination in the monkey. $\mathrm{J}$. Comp. Physiol. Psychol. 50: 630-635.

Woolsey, R. M., and J. S. Nelson (1975) A symptomatic destruction of the fornix in man. Arch. Neurol. 32: 566-568.

Wyss, J. M., L. W. Swanson, and W. M. Cowan (1979) Evidence for an input to the molecular layer and the stratum granulosum of the dentate gyrus from the supramammillary region of the hypothalamus. Anat. Embryol. (Berl.) 156: 165-176. 